\title{
Technical Horticulturists on the Internet
}

\author{
Laurence A. Sistrunk ${ }^{1}$
}

\begin{abstract}
Summary. H orticulturists need to stay abreast of the rapidly changing technology for disseminating research and teaching information. By connecting to the Internet and using the various tools available, horticulturists can gather information needed to communicate with colleagues and other clientele as a means of highlighting our science. The use of network software, such as FTP (file transfer protocol), G opher, Veronica, Archie, J ughead, WAIS (wide area information servers), M osaic, N etscape, Telnet, IRC (Internet relay chat), and WWW (World Wide Web) is essential in navigating the super information highway. This largely academically based network will evolve based on needs and politics in the years to come.
\end{abstract}

Additional IndeX words. FT P, G opher, E-mail, Veronica, Telnet, TC P/ IP

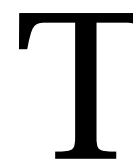

he Internet is a vast international network of networks that enables computers of all kinds to share services and communicate directly, as if they were part of one giant, seamless, global computing machine. It is comprised of thousands of separately administered networks of many sizes and types. Each of these networks is comprised of as many as tens of thousands of computers; the total number of individual users of the I nternet is now estimated at more than 8 million. On the World Wide Web (WWW) system, there were 24 million web pages with 12 billion words in April 1996. This high level of connectivity fosters an unparalleled degree of communication, collaboration, resource sharing, and information access. In the $U$ nited States, the $\mathrm{N}$ ational Science Foundation N etwork (N SFN et) comprises the I nternet backbone (a very high-speed network that connects key regions across the country). The N SF N et likely will evolve into the $\mathrm{N}$ ational Research and $\mathrm{Education} N$ etwork (NREN) as defined in the $\mathrm{H}$ igh- Performance Computing Act of 1991 (P.L. 102-194, signed into law by President Bush on $9 \mathrm{Dec}$. 1991) (L evine and Baroudi, 1993). Commercial services, such as CompuServe, Prodigy, America $\mathrm{O}$ nline, $\mathrm{G}$ enie, $\mathrm{M} \mathrm{Cl} \mathrm{M}$ ail, etc., provide access to the Internet for e-mail, mail servers, FTP, and Bitnet searches. For these services, no IP (Internet Protocol) address is used. 


\section{Internet services}

The following services are available to horticulturists on Internet:

- E-mail, which islike the post office (only faster).

- $\quad$ Talk, which is like the telephone (except that the user has to type out everything in a bulletin board format).

- Internet relay chat (IRC), which is like $C B$ radio-many users typing messages in real time.

- File transfer protocol (FTP), which isfor fetching programs and documents from remote computers.

- Telnet, which is used to operate those remote computers from a local desktop microcomputer.

- Archie, Veronica, Jughead, and WAIS (wide area information servers), which are tools for searching the huge libraries of information stored on the Internet (Levine and Baroudi, 1993; Willmott, 1995)

- Gopher, for tunneling quickly from one place on the I nternet to another.

- The World Wide Web, a more advanced navigation system that organizes its contents by subject matter.

- M osaic and N etscape, kinds of onscreen control panels that enables users to drive through the Web by pointing and clicking their electronic mouse. The latest language that builds interactive applications into proprietary browsers is called JAVA (N icholaisen, 1996). This new protocol will have evolved several times before this article is published.

- Internet Talk Radio, which broadcasts sound recordings (like the popular interview show "G eek of the Week")

- CUSeeM e, an Internet video conferencing system that enables up to eight users to see and hear each other on their computer screens

- U senet, a collection of electronic bulletin boards (called newsgroups) set up by subject matter and covering just about every conceivable topic, from molecular biology to nude sunbathing. The newsgroups are organized into hierarchies, such as science (SCI), recreation $(R E C)$, computers (COMP), society (SOC), and the miscellaneous category called alternate (ALT) (M cC arthy, 1995). A sampling includes sci.astro.hubbleastronomical datafrom theH ubbleSpace Telescope, rec.arts.books- where users gather to discuss their favorite authors, comp.risks-a digest of brief reports about computer bugs, and alt.best.of.internet-aplace wherepeople re-post choice tidbits found on the $\mathrm{N}$ et
- Software and graphics, literally thousands of scanned pictures, sounds, software and video clipsresiding on serversall over the world that we, as horticulturists can download to our local computers for use in classroom teaching and for research collaboration.

\section{Physical connections and logical conventions}

Connections can consist of any of a variety of communication media or methods: metal wires, microwavelinks, packet radio, or fiber-optic cables. These connections usually are established in areas or regions by the particular networking organization with authority or economic interest in that area. For example, a university academic department may lay E thernet cableto connect its personal computers and workstations into a local area network ( $L A N$ ), which then is connected to the cables the campus laid to connect its buildings together, which is then linked to cableslaid by a regional network, which itself ties into the N SFN et backbone, the infrastructurefor which wasfunded by the U nited States government. Therefore, the path between any two points on the Internet often traverses physical connections that are administered by a variety of independent authorities. The horticulturist can either hook in directly or, if no direct cable can be accessed with an Ethernet card, a modem connection must be used.

For various computers (from personal computers to mainframes) to communicate with other computers over a network, there must be conventions on how that should occur. These conventions are called communication protocols. At present, the Transmission Control Protocol/ Internet Protocol (TCP/IP) suite of protocols defines how Internet computers are to communicate. In the future, the 0 pen Systems Interconnection (OSI) suite of protocolspromulgated by the International Standards Organization (ISO ) may be supported on the Internet as well. These protocols define how certain applications are to be accomplished: electronic messaging, on-lineconnections, and thetransfer of files.

\section{Electronic mail (e-mail)}

E-mail is a fast, easy, and inexpensive way to communicate with other Internet users around the world (Frey and Adams, 1993). Internet users often find that the expanded capability to communicate with colleagues around the world leads to important new sources of information, collaboration, and professional development. In many instances, a reply is received within hours or even minutes. 
Besides basic correspondence between two network users, e-mail presents additional opportunities for communication. Through various methods for distributing e-mail messages to lists of subscribers, e-mail supports electronic discussions on a wide range of to pics. These discussions bring together likeminded individuals who use such forums for discussing common problems, sharing solutions, and arguing issues.

A nother type of electronic communication that is growing in popularity is the electronic journal (e-journal). Although some ejournals require certain types of software and hardware to display each issue, most are distributed to a list of subscribers as an e-mail text message, either complete as one issue or retrievable at the article level by mailing a command to a software program that automatically sends the appropriate file. The very definition of a journal is undergoing change in the electronic environment, as e-journal publishers experiment with different publication models (e.g., sending articles out individually as soon as they are ready rather than waiting until a group of articles are gathered for an issue).

\section{Remote login}

Remotelogin istheability of acomputer user in one location to establish an online connection with another computer elsewhere (Tennant and Lipow, 1993). O nce a connection is established with a remote computer, users can use their remote systems as if their computer were ahard-wired terminal of their system. Within the TCP/ I P protocol suite, this facility is called T elnet. $U$ sing T elnet, an Internet user can establish connections with a multitude of bibliographic databases (primarily library catalogs), campus information systems of various universities, full-text databases, datafiles (e.g., statistics, oceanographic data, meteorologic data, geographic data, etc.), and other online services. $M$ any of these systems are available for any I nternet user to access and use without an account.

Internet users can connect to systems on the other side of the globe as easily as (and generally not much slower than) they can connect to a system in the next building. In addition, since many Internet users are not currently charged for their network use by their institutions, or at least are not charged by the level of their use, cost is often not a significant inhibitor of usage. Therefore, the barriers of distance, time, and cost, which are often significant when using other forms of electronic communication, can be reduced in the Internet environment. A compensating disadvantage is that initial costs for I nternet connection can be high, and access can be technically demanding.

\section{How do horticulturists get connected?}

That depends on how connected a user wants to be.

- If the user has an account at CompuServe or Prodigy, he or she can already send and receive e-mail through the Internet.

- If the user has an America O nL ine account, he or she al so can use other I nternet services, like the electronic bulletin boards (called newsgroups) and browse the WWW with a graphical interface.

- If the user has an account at Delphi or any one of dozens of smaller commercial operations, access can be gained to even moreof the I nternet-butstill indirectly, through a dial-up modem.

- For a user to be directly plugged into the Internet and use all its services, the computer must have what is called a TC P/ I P (for Transmission Control Protocol/ Internet Protocol) connection. To set that up, most users would probably need an Ethernet card and a technical computer person. Typically a hard-wired connection will be made with a thin-wire or a twisted-pair cable. Each has its own type of connectors. Combination Ethernet cards come with both types of connectors. If the PC (personal computer) isnot within the proximity of the server for hard wiring, it is still possible to have completeaccesswith PPP (Point-to-Point Protocol) or SLIP (Serial Line Internet Protocol). These are dial in ports to the main server, which currently use 14,400 and 28,800 baud. These are significantly slower than thehard-wired speed of transfer, but many functions can be accessed at a slower speed, especially if it does not require heavy file transfer.

\section{FTP}

A nother application of the Internet is the ability to transfer files from one I nternetconnected computer to another. This function is provided by theFile T ransfer Protocol (FTP) of the TCP/ I P protocol suite (L evine and Baroudi, 1993). In a method similar to using T elnet, network users initiate an online connection with another Internet computer via FTP. But unlike Telnet, this online connection can perform only functions related to locating and transferring files. This includes the ability to change directories, list files, retrieve files, etc.

Types of files that can be transferred using FTP include virtually every kind of file that can be stored on a computer: text files, software programs, graphic images, sounds, files formatted for particular software programs (e.g., files with word processing for- 
matting instructions), and others. $M$ any computer administrators have set aside portions of their machines to offer files for anyone on the Internet to retrieve. These archive sites support anonymous logins that do not require an account to access, and therefore are called anonymous FTP sites. The user types in the word "anonymous" as the user id for the foreign host. The foreign host will request a password, and the user will type the mainframe account user id for the password. To locate files, Internet users can use the Archie service, which indexes files from more than 900 separate anonymous FTP sites (Tennant, 1993).

$M$ any computers on the Internet have collections of software and useful information that areavailableto thenetwork community via programs that follow FTP. U sing FTP often seemsalittle basic and unnecessarily complex to the horticulturist. M ore userfriendly software like M osaic or N etscape handle the tasks of moving files with mouse point-and-click movements rather than keyboard commands.

U nfortunately, there isno way, using FTP, to determine beforehand, that a certain fileis not a text-only file. So while Gopher may display the names of such documents in listings, and even let the user attempt to retrieve them, it ultimately will realize that such filesare not universally useful or understandable-and it will refuse the request when it realizes that it isin the process of retrieving a binary file. Sorry. That's the best that our helpful Gophers can do. Well, actuallythisisnot completely true. $M$ acintosh software and documents (other than text files) commonly areencoded into text filesfor transmission over the network. A common standard for such encoding is referred to as BinH ex. The M acintosh Gopher client will recognize such files and allow you to fetch $B$ in $H$ ex encoded $M$ acintosh applicationsand documents. The files will be decoded for use automatically. Such files may not be accessible from PC or UNIX clients. The user usually can tell that a file contains a BinH exencoded $M$ ac fileby the fact that the filename ends in ".hqx".

$\mathrm{N}$ ot every horticulturist has access to a newer computer with a graphical interface, but even terminalsor older computers may be used for transferring files to the mainframe computer account through an educational institution. After logging on to one mainframe, the user can connect to the second mainframeby typing: O PE $\mathbf{N} \varangle$ Return $>$. Then the address of the FTP site is entered, for instance: ftp.wustl.edu. Press the $\varangle$ Return $>$ or $\varangle$ nter $>$ key. N ow theuser may log on to the other mainframe. If the system promptsfor the user id, type:<userid > $\varangle$ Return> Example: abc1234 ४Return>. <password> $\varangle$ Return>. Example: hort95 $\varangle$ Return $>$. To get a listing of the files on the account to which the user is FTPing to, type: LS $\varangle$ Return $>$.

FTP transfers files in ASCII format or Binary format. The default format is ASCII. To change to binary format before transferring the file, type BIN $<$ Return> To change to ASCII format before typing the file, type: ASCII <R eturn>

To transfer a file from the remote site to the local system, type: GET <foreignfile> $\varangle$ Return $>$

To transfer a file from the local system to the remote site, type: PU $\mathbf{T}<$ localfile $<R e$ turn>.

To transfer a group of files from the remote site to the local system, type: MGET $<$ foreignfile> <foreignfile>... [(Replace] $\varangle$ Return $>$.

\section{Type name action When selected}

Text file
Binary file
Directory
Search item
Phone book
Terminal session
Error

Retrieves and displays the file

Retrieves the file

Shows you more items

Prompts for text to search for

Prompts for person to look up

Connects you to a non-G opher service None

To transfer a group of files from the local system to the remote site, type: M PU T $<$ localfile> <localfile>... [(R eplace] $\varangle$ Return $>$

On the remote site, it is possible to change directories by entering $\mathbf{c d}<$ new directory name $>$ to enter another subdirectory. To enter a subdirectory one level up, enter CD U P or CD ..., depending on what system is being accessed. Typically, a directory called "pub" is where the interesting things are stored. Some sites will have a file with a name like Is-IR, that contains a complete list of the files on that site. $O$ therwise, the user types IsIR and getssuch alisting-for somesites, this can take a long time. After the remote system has changed directories, type DIR again to see the contents of the subdirectory. Keep moving down through the directories until the desired file is found.

U sually, filesaregrouped in archivefiles, so the horticulturist doesn't have to get many small files separately. The most common archival file format for the Internet is "tar". O ccasionally, programmers use "shell" archives (shar) instead. The tar archives are unpacked by running the tar command-the user first givesthe "tart" command on thefile to see what it contains, before unpacking it. 
"Gopher

allows users to

access various

types of

information

residing on

multiple

computersin

a seamless

fashion"
U sersunpack "shell" archives by first running them through the Bourne "shell" to unpack them. (T hesimplest way isto use the "unshar" command)

Files often are stored compressed-for UNIX, the most common scheme is the compress program, indicated by a.Z suffix on the file name. Sometimes, sysops (system operators) use programs like arc or zoo, which are combined archival and compression formats. (There are probably other archival formats as well-talk to the systems staff if they are encountered for instructions on how to deal with them).

When retrieving nontext files, the user must use binary mode, otherwise the file gets messed up. The user first inputs the binary command. (It's safe to set this for text files. If the site at the other end is non-UNIX, the user selects some other mode- see the documentation for that site and for FTP)

\section{Common file types}

These are the common U N IX file types: Suffix FTP Type, .Z bin compress, , arc bin ARChive, shar ascii SH ell AR chive, .tar bin TapeAR chive, .uu ascii uuencode/ uudecode, and.$z 00$ bin Zoo

There are more file compression types than these, so some investigation on file types may be necessary if other suffixes are encountered.

Exiting FTP automatically closes the connection to the remote system. The user types Q U IT and presses <Return $>$ to exit FTP. The visible system prompt should return. The user then checks the local directory to make sure the files were transferred.

\section{Extended services}

The three basic Internet applications, electronic mail, remote login, and file transfer, are also building blocks of more sophisticated applications that usually offer increased functionality and ease of network use. Tools such as Gopher, WAIS, and WWW go beyond the three basic Internet functions to make information on the network easier to locate and use. Gopher is a project of the $U$ niv. of M innesota that uses a series of menus to organize and automate access to information and other on-line systems wherever they reside on the Internet. The Wide Area Information Servers (WAIS) project of Thinking $M$ achines, Apple Computer, D ow J ones\& C 0 ., and K PM G Peat M arwick, seeks to providea common interface to a multitude of Internet databases. WWW is a hypertext interface to Internet information resources that was developed at CERN in Switzerland (Tennant, 1993). The trend toward more powerful, user-friendly networked information resource access systems is likely to continue as the Internet grows and matures.

\section{Gopher}

The Internet Gopher is a distributed document delivery service (Levine and Baroudi, 1993). It allows users to access various types of information residing on multiple computers in a seamless fashion. In other words, users can access information from all over the world without having to know a specific machine name or how to communicatewith it. Thisisaccomplished by presenting the user a hierarchy of menus where each menu entry provides access to text files, directories, CSO N ameservers, other gophers, etc., each of which can reside on any computer on the I nternet. U sers also can ask index servers to return a list of all documents that contain one or more words. Since an index server does full-text searches, every word in every document is a keyword.

The G opher software uses a client-server communications model. A Gopher Server is implemented on a host offering information to the rest of the Gopher world. It accepts simple queries and responds by sending the client a document. A Gopher Client enables users to communicate with Gopher Servers on the Internet network. A Client's view of the world is solely dependent on the default Server to which it connects. Through the Gopher Client, a user can select and request any of the information provided by a Server.

To enter the I nternet Gopher software, the userstype G O PH E R from the command prompt. This will bring up the main menu if they are accessing it through a mainframe. The list will look something like this:

$\varangle \mathrm{F}>$ About I nternet Gopher

$\varangle B>$ G IF picture of J oey R amone

$\varangle$ > Really I nteresting Administrative I nformation

$\langle\mathrm{P}>\mathrm{U}$ of MN Phone Book

$\langle>$ Search Pyrotechnics Recipes

$<\mathrm{T}>\mathrm{C}$ ard $\mathrm{C}$ atalog via Telnet

Any item on the list can be selected either by moving the selection bar with the arrow keysand hitting E N TER, or by doubleclicking with the mouse.

\section{Gopher item types}

At the beginning of each line in the item list is a character enclosed in brackets; this character tells the user what the item is. Gopher knowsabout thefollowing item types:

\section{Text files}

In the above example, line one refers to a filecalled "A bout I nternet Gopher". To see the contents of this file, the user selects the item with either the mouse or the keyboard. Gopher will attempt to retrieve the file, and display it in a separate window. If the file is too large for Gopher to fit into available 
memory, it will put up a dialog box requesting that the user specifies a file name and directory in which to save the file in. The file then is opened with "a favorite word processor" to see the contents.

The user also may choose to save the information as afile, even if it issmall enough for Gopher to display immediately in a window. The usual procedure is to choose Save File... from the File menu, and enter the file name and directory that the file should reside in.

\section{Binary files}

The second item in the list above is a binary file, a GIF picture of one of the programmers. Choosing this item will cause a dialog box to pop up, prompting for a file name in which to save the picture. O nce a file name has been entered and the OK button has been chosen, G opher will retrievethe file. Instead of displaying the file in a window, Gopher will show a progress indicator, so the user can tell when the file has been retrieved. To view the file, the user is required to quit Gopher and run a separate program capable of displaying GIF pictures.

\section{Directories}

The third item in our example listing is a directory. Directories are like folders or drawers full of other items. When Gopher first connects to the Gopher server that is specified as the starting point, the list that is displayed shows the contents of the main or "root" directory of that server.

To see the contents of a directory, it is selected from the list using either the mouse or the keyboard. Gopher will request a list of the contents of the subdirectory that was selected, and will present thislist in thebrowser window.

If users anticipate returning to a particular directory on a particular server, they can use the Bookmark L ist to save that position in G opherSpace. To save abookmark, users first navigate to the place to remember, and then choose "Open Bookmark File" from the "File" menu. O nce the bookmark list opens, they click on the "Add" button and are prompted for a name for the bookmark. To return to the bookmarked position later, they simply open thebookmark list and choose the bookmark to return to.

\section{Phone book items}

The fourth item in the example is a Phone Book item. Phone Book items are pointers to databases that contain information about people's phone numbers, office locations, e-mail addresses, and so on; these databasesarecalled CSO or PH servers. When a Phone Book item is selected, a dialog box will pop up, and Gopher will ask the database server for a list of items. O nce the list of these itemsisbuilt, theuserscan lookup a person by typing hisor her nameinto the dialog box and choosing the "Lookup" button. Any matching items will be displayed in a separate window, and can be saved to disk using the File menu's Save File command.

If more information is desired, to narrow the search, the user can choose the "M ore Choices..." button, and be presented with a larger dialog box, containing a list of fields which can be used in a query. The user can build a query by selecting an item from the list, and typing in the search key next to the item name when it appears on the screen. $\mathrm{Q}$ ueries can consist of up to five items; the query is changed, by selecting the "Clear" button to clear all of the chosen items. Once the query is set up, the "Lookup" button is selected, and the query will be sent to the server.

\section{Search items}

$\mathrm{N}$ ext in the listing is a Search item for an interesting set of recipes. A search item is a computer that can search various gopher servers for files containing text that the user specifies. The user selects a search item, and a dialog box appears in which the search text is entered. To start the search, the user clicks the computer pointing device on the "Search" button, or just presses the $\varangle$ Enter $>$ key.

The search item returns a list of files that it finds and which contains all of the text that is entered in the input box. If the word "hybrid" is used, the search item would return a list of all of the files containing that word. If "hybrid spinach" is the search keywords, the search item will return alist of files that contain the word "hybrid" and the word "spinach"; it would N O T return thenames of files that had only one of the two words.

\section{Search items and logical operators}

Logical operators are special words that allow the user to set up specific search conditions. The logical operators "and", "or", and "not" are supported by search items. O ne or more of these operators is entered to narrow the field of the search.

There are three different types of search items in G opherSpace. The first type assumes an implicit "and" between words, and will allow the use of logical operators to change this behavior. Thesecond assumes an implicit "or" between words, and also will allow the use of logical operators to alter the default. The third assumes "or", and ignores logical operators. U nfortunately, the only way to find out which type of search item is being used is to experiment (unless it is labeled

"Logical
operatorsare
special words
that allow the
user to set up
specific search
conditions"


"M ost

Veronica-

access menus

offer several

types of

searches" explicitly as one of these three types).

For example, hypothetically asearch item is found that assumes "and" between words, and also understands logical operators. Suppose the user wants to find files that contain theword "carrot" or theword "spinach", but not both. This can be done by entering "carrot or spinach" in the input box. The word "and" also could be used to explicitly indicate that the files are being searched and must contain both words; this allows the user to set up specific search conditions by using multiple logical operators. L ogical operators are evaluated in right-to-left order; the search pattern "spinach and carrot or broccoli" would result in a list of files that contained the word spinach and the word carrot, or just the word broccoli.

To exclude words from the search pattern, the user enters "not". The search phrase "M innesota not Texas" would produce a list of files containing at least one instance of the word "M innesota", and no instances of the word "Texas".

Any query can becanceled at any time. If a user wants to cancel aquery and throw away any directory listing or text that has been received to this point in the search, the window that is associated with the query can be closed. To cancel a query without closing its window, the user types Alt-Z or chooses the "Alt-Z Cancel Query" hotspot in the status line.

\section{Veronica}

Veronica is an acronym for very easy rodent-oriented net-wide index of computerized archives; believe it or not (Zabinski, 1995). This index and retrieval system can locate items on most of the $G$ opher servers in the Internet. The Veronica index, at one time, contained about 10 million items from approximately 5500 Gopher servers (J une 1994). Information on Veronica was obtained from gopher:// veronica.scs. unr.edu:70/ 00/ veronica/ how-to-queryveronica.

Veronica finds resources by searching for WO RD S in TITLES. It doesnot do afulltext search of the contents of the resources; it finds resources with titles containing specified search word(s). The "title" is the title of the resource as it appears on the menu of its H OM E gopher server.

Veronica is used with a Gopher client. Theclient chooses "Veronica" from themenu of some Gopher server, and enters a set of query words or special directives. When the search is finished, the results are presented as anormal Gopher menu. The user may browse the discovered resources in this menu, just as easily as any other Gopher menu.

M ost Veronica-access menus offer sev- eral types of searches. In addition to these predefined types, the user can compose Veronica queries using a number of special options to focus the search more precisely. These options should be used when appropriate, as they will make it much easier to locate resources.

$M$ any $V$ eronica-access menus offer a list of various Veronica server sites; in this case the user will have to choose a server site. I deally, it does not matter which server is used, as all servers will give the same answers. In practice, all the servers do not update the index at the same time, so there will be some difference in the results. Some servers will return an answer faster than others, depending on load and network traffic.

M ost access menus offer two predefined search types:

1) > Search GopherSpace by keywords in Titles

This search will find ALL TYPES of resources whose titles contain the specified search words. The resources may be of any Gopher data TYPE; e.g., ascii documents, Gopher directories, image files, binary files, etc.

2) > Search Gopher DIRECTORIES O N LY for keywords in Titles

This search will find only Gopher DIRECTO RIES whose titles contain the specified words. This search can be very useful to find only major holdings of information which relate to the query. After Veronica finds the Gopher directories, any of them can beopened to see the contents in more detail. This is especially useful to avoid being overwhelmed by too many results, if searching with a common word such as " university" or "internet".

A personalized query can be defined, specifying only certain TYPES of gopher resources, by using the -t option. For instance, ONLY image files will be found by including the phrase "-tl" in a query. For more about " $t$ " limiters, users should read the instructions on-line at gopher:/ / futique.scs.unr.edu:70/ 00/ veronica/ howto-query-veronica or have someone download it for them. When a query type is selected, the G opher client will present a dialog box. $\mathrm{H}$ ere the users enter the query words. The search is not case-sensitive.

Better results may be obtained by entering a multiple-word query rather than asingle word. Multiple word queries will find only those titles containing all the specified words. For instance, "horticulture" may find 522 items; but "garden vegetables" may find 12 items. The user should be as specific as possible. It also helps to be imaginative. Consider how gophers are organized; the information wanted may not be found under "horticulture", but under the more general 
heading of "plants" . A multiple-word query does not require that the words be adjacent in the title, or that they appear in any particular order. The query "perennial grasses" will locate the same items as "grasses perennial".

The search understands the logical operators AND, NOT, and OR. If a simple multiple-word query is used, it is the same as using AN D between the words. For instance "annual flowers" is the samequery as "annual and flowers". U sers input AND to create a tightly focused query.

An asterisk (*) at the TRAILIN G END of a query word will match anything. $U$ seit as alimited form of wildcard search. The asterisk character may be used ONLY at the end of words; the search will fail if an asterisk is placed within a word or at the beginning of a word.

Search words must be at least two characters long. Shorter words will be ignored. 0 ther techniques are discussed in some of the help files that can be downloaded from the $M$ innesota gopher.msu.edu or from certain Internet resources (Levine and Baroudi, 1993).
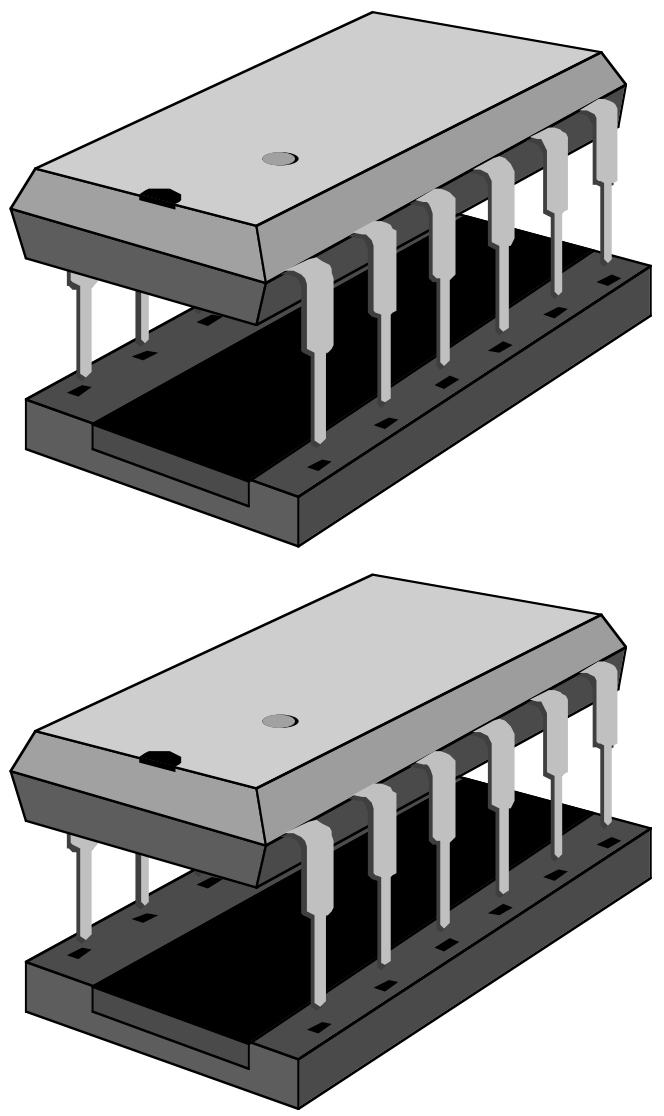

\section{Literature Cited}

Andrews, D., C. Brett, S. Gonzalez, M. R oszowski, G. R owe and D. Berlind. 1995. E-mail for the purists. Windows Sources 3(11):73-78.

Frey, D. and R. A dams. 1993. A dictionary of electronic mail addressing and networks. 2 nd ed. 0 'Reilly and Associates, Inc., Sebastopol, Calif.

Levine, J and C. Baroudi. 1993. The Internet for dummies. ID G BooksWorldwide, Inc., San M ateo, Calif.

M cC arthy, S.P. 1995. U seN et groups you can join to find the hidden good stuff. G overnment $\mathrm{Com}$ puter $\mathrm{N}$ ews 14(24):38.

Nicolaisen, N. 1996. Waking up the Web: Sun M icrosystems' J ava gives programming on theW $\mathrm{eb}$ a boost. Computer Shopper. 16(2):581-582.

Tennant, R ., J. O ber, and A .G. Li pow. 1993. C rossing the Internet threshold: An instructional handbook. Library Solutions Press, Berkeley, Calif.

Willmott, D. 1995. The Internet: $M$ ake it work for you. PC M ag. 14(17):106-107.

Zabinski, T. 1995. Surfing with Veronica: Thisfree (but sometimes frustrating) resource can help you search thel nternet. Electronic L earning 15(3):17.

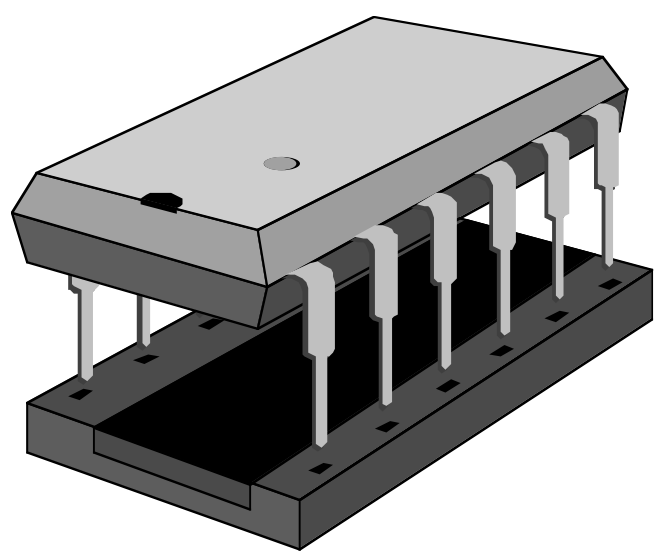

